\title{
Preface to the special issue on "Mechanics of Fibre-Reinforced Materials: Theory and Applications, Part II"
}

\author{
K. P. Soldatos · R. W. Ogden · J. Merodio
}

Received: 22 January 2015 / Accepted: 26 February 2015 / Published online: 8 April 2015

(C) Springer Science+Business Media Dordrecht 2015

This special edition of the Journal of Engineering Mathematics (J Eng Math) is a continuation of the ongoing effort to promote quality research in the subject of the Mechanics of Fibre-reinforced Materials (FRMs) on an international scale. This effort started with a special issue of this journal on the subject, published in 2010 and edited by two of the present co-guest editors [1], and continued more recently with the organization of the 551st Colloquium of the European Mechanics Society (Euromech 551, 2-5 September, 2013, University of Nottingham), which was co-chaired by all three editors of the present volume. The title of the Euromech Colloquium 551 was identical to that of [1]. The present volume comprises nineteen research papers that were presented in the Colloquium, and an additional paper (Paetsch and Dorfmann) invited by virtue of the contribution that one of its co-authors had published previously in [1]. All twenty papers were submitted to J Eng Math after the end of the Euromech 551 and passed successfully through the standard peer review process of this journal. The present special issue is thus a natural continuation of the earlier edition [1].

The preface of [1] outlined briefly the history of the nonlinear theory of FRMs and acknowledged the profound role that a group of Nottingham University mathematicians, led for long time by A. J. M. Spencer, played in its development. It described qualitatively each of the seven publications contained in [1] and highlighted research trends which were expected to influence the subject of the Mechanics of Fibre-reinforced Materials in the long term. Those trends remain highly relevant and need not be repeated in the present volume, the size of which is almost three times the size of its predecessor. Such a successful continuation of [1] makes it impractical to give a detailed description of the twenty high-quality papers contained in the present issue, but some general observations and research trends are now highlighted.

K. P. Soldatos $(\varangle)$

School of Mathematical Sciences, University of Nottingham,

University Park, Nottingham NG7 2RD, UK

e-mail: kostas.soldatos@nottingham.ac.uk

R. W. Ogden

School of Mathematics and Statistics, University of Glasgow,

Glasgow G12 8QW, UK

e-mail: raymond.ogden@glasgow.ac.uk

J. Merodio

Department of Continuum Mechanics and Structures, Escuela de Caminos, Canales y Puertos, Universidad Politécnica de Madrid, 28040 Madrid, Spain

e-mail: merodioj@gmail.com 
Most of these observations and trends are principally related to and highlight the multidisciplinary nature of the research conducted in FRM mechanics. They also reveal the multidisciplinary nature of the skills and knowledge required of colleagues who are engaged in FRM research. For instance, several of the publications included in this issue (e.g. Benaarbia et al.; Gao et al.; Selvadurai; Soldatos; Topol et al.) relate directly to experimental observation and applied mechanics. Nevertheless, it is also evident that all authors share the belief that mathematical modelling and analysis form a natural language for the description, understanding and interpretation of the real-life behaviour of FRMs.

The theoretical mechanics content of the research described in more than half of the papers included in this special issue is based on theoretical modelling and concepts associated with the nonlinear elastic behaviour of FRMs (Dagher and Soldatos; Destrade et al.; Domanski; Gao et al.; Gower; Guz et al.; Hamdaoui and Merodio; Huang et al.; Paetsch and Dorfmann; Topol et al.; Wang et al.), while a similarly large proportion of papers employ linear or linearized elasticity concepts (Domanski; Guz et al.; Huang et al.; Knops; Lurie et al.; Milosavljevic et al.; Paetsch and Dorfmann; Parnell and Calvo-Jurado; Selvadurai; Vollmecke et al.). There are also a number of papers that deal with or refer to non-elastic behaviour and failure of FRMs (Benaarbia et al.; Guz et al.; Huang et al.; Lurie et al.; Soldatos), while studies of and references to failure mechanisms and modes in elastically behaving FRMs are also well represented (Guz et al.; Hamdaoui and Merodio; Milosavljevic et al.; Paetsch and Dorfmann; Selvadurai; Vollmecke et al.; Wang et al.).

It is also observed that the research described in at least a third of the papers is motivated by applications that FRM mechanics meets in biological- and bioengineering-related problems (Destrade et al.; Gao et al.; Gower; Huang et al.; Paetsch and Dorfmann; Topol et al.; Wang et al.). Attention and/or reference is also given to a number of more classical topics of FRM application, such as wave propagation (Domanski; Milosavljevic et al.; Paetsch and Dorfmann), thermo-mechanics (Benaarbia et al.; Knops) and material characterization (Gao et al.; Guz et al.; Li et al.; Lurie et al.). Particular mention should be made of the fact that, after one and a half centuries of their involvement in and application to common engineering applications, the fundamental theoretical concepts of Saint Venant's principle are still being examined and developed (Knops). Studies on more recent but equally classic topics, such as material homogenization (Parnell and Calvo-Jurado) and fibre-scale (Huang et al.; Lurie et al.; Soldatos) or polar material behaviour (Soldatos), are also present.

There is also clear evidence (e.g. Huang et al.; Li et al.; Wang et al.) that, due to the mathematical complexity involved in the aforementioned research topics, confrontation of problems related to more advanced material configurations and/or more advanced physical difficulty may require the use of purely computational methods. Still though, a considerable number of papers published in this issue deal with fundamental theoretical developments which rely on the application of suitable mathematical approaches and methods (e.g. Dagher and Soldatos; Destrade et al.; Domanski; Gower; Hamdaoui and Merodio; Knops; Lurie et al.; Parnell and Calvo-Jurado; Soldatos; Topol et al.). The use of material constraints (e.g. Dagher and Soldatos; Gower; Guz et al.; Milosavljevic et al.; Paetsch and Dorfmann; Soldatos) is of great help in these analytical endeavours. However, wide appreciation of the significance of the latter becomes usually possible through the study, interpretation and meaningful presentation of numerical results, which is not possible without the development of modern computational approaches. Nevertheless, numerical results obtained on the basis of analytically motivated developments are also expected to become benchmarks that will assist verification and further successful exploitation of computational methods.

A final comment of great significance stems from the fact that more than two-thirds of the papers published in this issue, namely all those based on linear and/or nonlinear elasticity considerations, are heavily based on the concept of the "strain energy function/density" that was introduced by George Green. In fact, even publications which consider non-elastic FRM behaviour refer to posterior similar concepts, such as the "Helmholtz free energy" or the "thermodynamic potential", the "dissipation potential", and the "yield function" or the "plastic potential". These concepts have been, and are still of profound importance and influence in our efforts to comprehend and to model material behaviour, in general, and FRM behaviour in particular. Studies that aim to identify the strain energy density characteristics of particular materials met in nature, such as in the study described by Gao et al., are thus particularly welcome. 


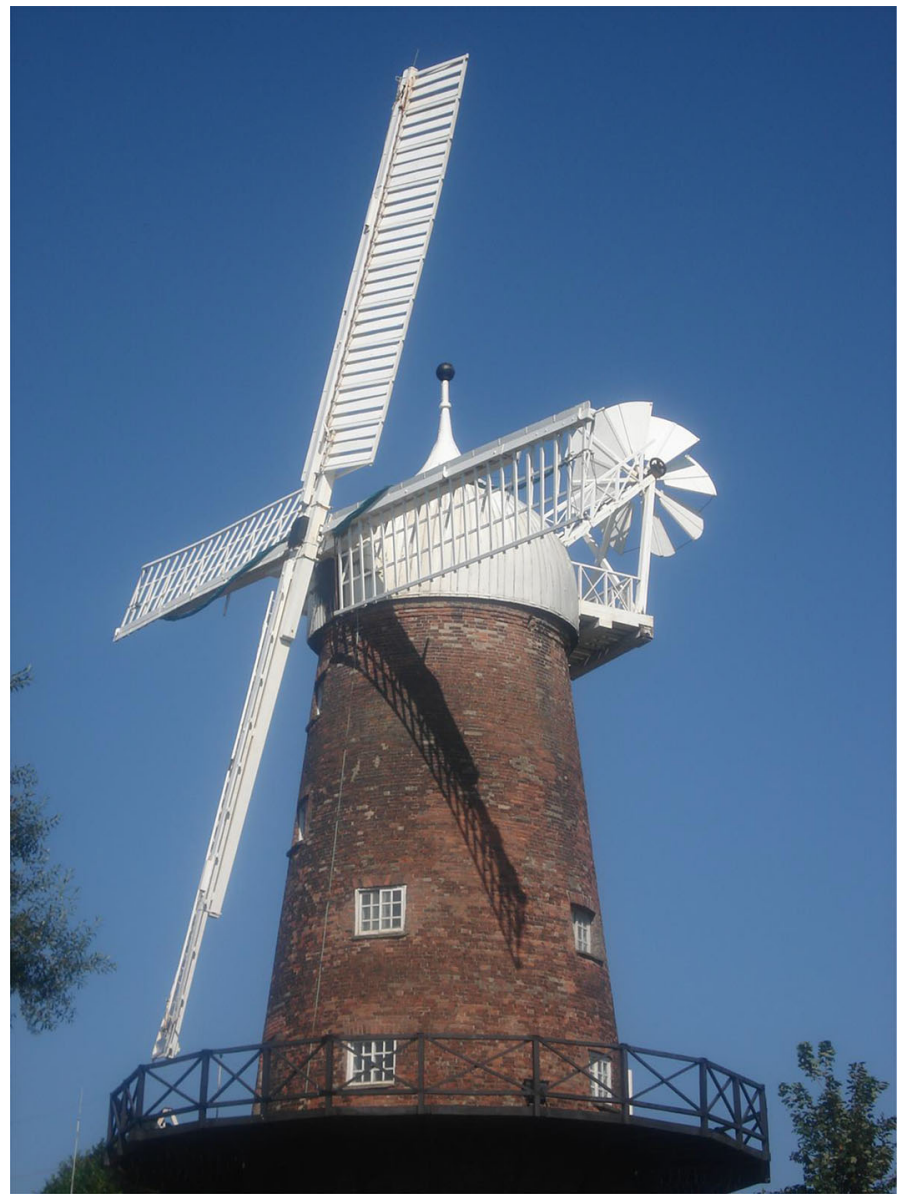

Fig. 1 George Green's Windmill at Sneinton, Nottingham, in a sunny morning of August 2012

It is pointed out in this connection that, after essentially repeating the FRM historical briefing outlined in the preface of part I [1], the booklet of abstracts of the Euromech Colloquium 551 paid tribute to George Green's profound contributions by adding the following couple of paragraphs:

"Nottingham was also the birthplace as well as the principal workplace and residence of George Green, the famous 19th century mathematician. Widely known for his famous theorem, his involvement with the theory of electromagnetism and the introduction of the now-called "Green's functions", George Green was also the first to define an elastic material as one for which a strain-energy function exists. He thus gave rise to the part of solid mechanics which is now known as Green elasticity or hyperelasticity. An alternative route of historical connection is thus emerging between Nottingham and the theory of fibre-reinforced materials, which was initially introduced as a continuum mechanics theory of hyperelastic solids.

Notably, George Green worked also as a miller in Sneinton, Nottingham. The "Green's Windmill”, built and owned initially by George's father, was completely restored in 1988 through the combined initiatives of the City of Nottingham and the University of Nottingham. It is now fully functioning and can produce again and even sell flour. It is visible from the higher grounds of the University Park and, together with the small museum, built in the proximity of the mill, honours the memory and achievements of the great mathematician".

It is thus fitting to conclude this short preface by adding that, through recorded actions of its head, A. J. M. Spencer, the former Department of Theoretical Mechanics at Nottingham supported overwhelmingly the University driving 
forces that fought for and, finally, succeeded and achieved the restoration of Green's Windmill (Fig. 1). This fact is partially recorded in the programme of a relevant two-day meeting (15-16 July, 1988) which was organized in the University Park under the title "George Green, Miller, Physicist and Mathematician". That programme contains a 45 minute presentation by A. J. M. Spencer, entitled "Green's Essay and the basis of the Theory of Elasticity". For personal purposes and reasons, Tony produced at the time a summary of his presentation, which he kept unpublished. There is though evidence that, upon written request received after the meeting, he posted a copy of that summary to one of the meeting participants. The present preface is followed by that unpublished summary of Tony's presentation, reproduced here with permission of his family.

All twenty papers that comprise this special issue appear afterwards in the order that they were submitted to the Journal of Engineering Mathematics for peer review evaluation.

\section{Reference}

1. Soldatos KP, Ogden RW (2010) Mechanics of fibre-reinforced materials: theory and application. J Eng Math 68(1):57-83 